\title{
REVIEW
}

For reprint orders, please contact: reprints@futuremedicine.com

\section{Alterations in the expression and activity of pre-mRNA splicing factors in hepatocarcinogenesis}

\author{
Carmen Berasain ${ }^{1,2}$, María Elizalde ${ }^{1,2}$, Raquel Urtasun 1,2, Josefa Castillo1, \\ Oihane García-Irigoyen ${ }^{1,2}$, Iker Uriarte ${ }^{1,2}$, Maria U Latasa ${ }^{1,2}$, Jesús Prieto ${ }^{1,2}$ \\ \& Matías A Avila*1,2
}
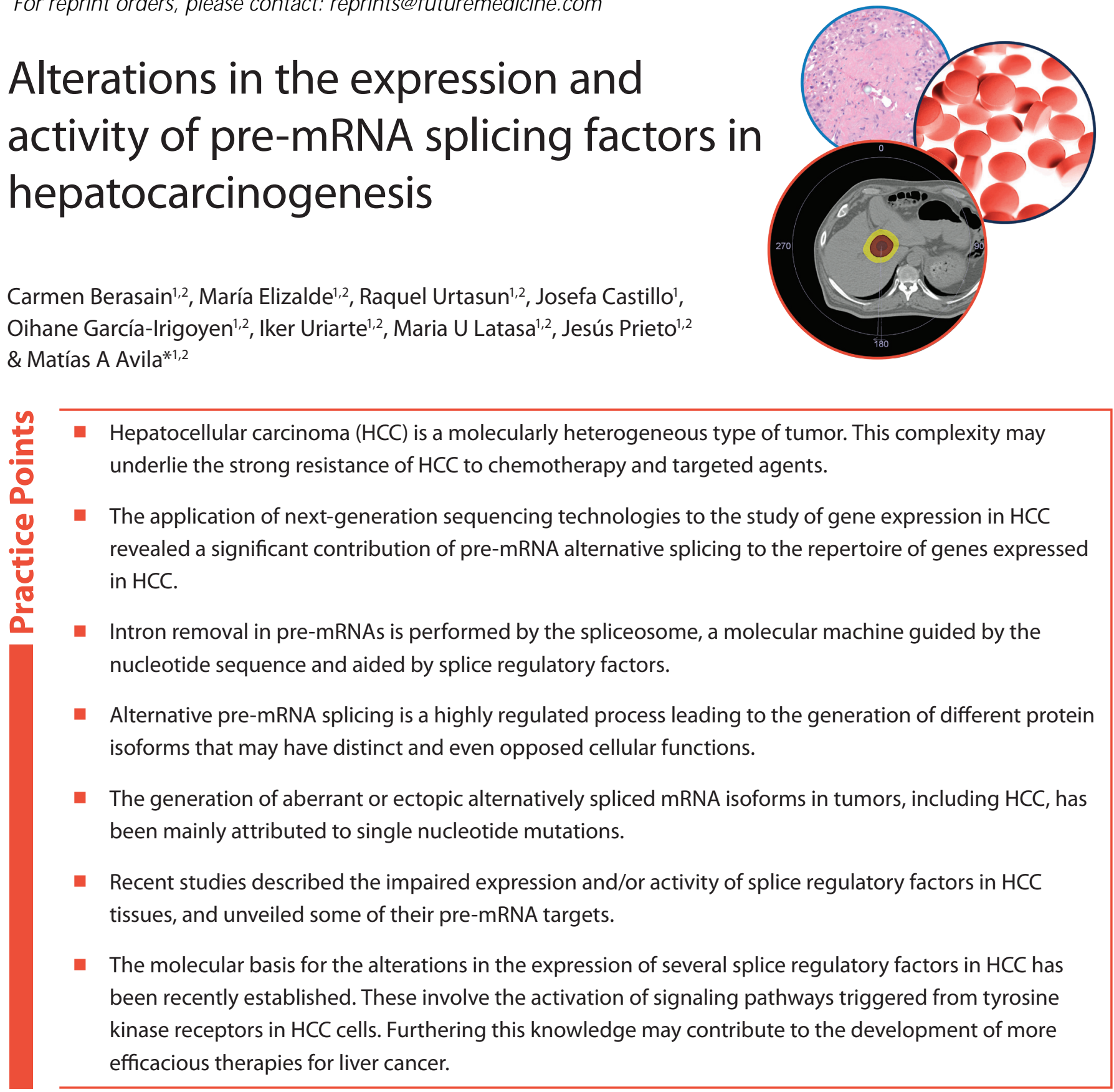

SUMMARY Hepatocellular carcinoma (HCC) is a molecularly complex tumor that is resistant to standard and targeted therapies, and thus a deadly disease. In this context, the identification of key alterations driving HCC development is therefore essential. The implementation of next-generation sequencing techniques has underscored earlier realizations of the marked dysregulation of pre-mRNA splicing in HCC. Impairments in alternative splicing may lead to the expression of protumorigenic protein isoforms and to the generation of unstable mRNA species. Mechanistically, mutations in key nucleotides are

'Division of Hepatology \& Gene Therapy, Centro de Investigación Médica Aplicada (CIMA), Universidad de Navarra, CIMA, Avda, Pio XII, n55, 31008 Pamplona, Spain

Future : Medicine ${ }_{\text {part of }}$ 
responsible for many of these alterations in different types of tumors. However, changes in the expression of factors involved in the regulation of the splicing machinery are also important determinants in the derangement of pre-mRNA splicing. Here we discuss recent reports on the alteration of splicing factors in HCC, the pathological significance of these changes, and the identification of cell signaling pathways leading to the missplicing of genes in hepatocarcinogenesis.

Hepatocellular carcinoma (HCC), the most frequent type of liver cancer, is often a fatal disease. HCC normally develops on a background of chronic liver injury and inflammation, most cases $(>90 \%)$ being associated with liver cirrhosis, a clinical condition that is the consequence of persistent liver tissue damage and the ensuing exacerbated regenerative/reparative response [1]. Another characteristic of the cirrhotic liver is the progressive loss of organ function, which is not only attributable to the reduced number of hepatocytes but also to changes in their gene expression profile towards a less differentiated hepatic phenotype [2-4]. Therefore, the chronically injured and cirrhotic liver is viewed as a preneoplastic condition in the multistep process of liver cancer development. The multifaceted natural history of HCC is paralleled by the complex molecular profile of this type of tumor [4-8]. Such complexity is to a great extent responsible for the high resistance to standard chemotherapy displayed by HCC [7]. Patients are often left with few therapeutic options that, depending on the size and number of the lesions, and the preservation of liver function, may include tumor resection, liver transplantation and locoregional therapies [9]. Additionally, the fact that cancer develops on a damaged organ further complicates the use of systemic agents due to increased risk of toxicity [9].

As demonstrated in other types of tumors, basic studies on the nature of the molecular alterations present in HCC cells indicated the existence of protumorigenic pathways that could drive the proliferation and survival of cancer cells [10]. Ideally, interfering with the activity of key elements within these pathways should quell cancer cell growth. The proof of principle that such targeted agents may be useful in treating HCC was the SHARP study [10]. In this trial the multitargeted kinase inhibitor sorafenib showed efficacy and significantly increased survival times in HCC patients by 3 months [9]. Although these findings were encouraging, sorafenib is the only targeted therapy that among over other 50 agents has shown some efficacy in HCC treatment so far [10]. This precarious situation has triggered an active search for additional molecular alterations that could constitute new therapeutic targets in HCC. High-throughput screens for differentially expressed genes using a variety of technologies, mostly microarray-based techniques, allowed the identification of several molecular pathways commonly dysregulated in HCC. These valuable studies revealed the existence of prognostic signatures in HCC tissues $[7,8]$, highlighted the predictive value of a gene expression signature in the peritumoral cirrhotic liver tissues [4], and contributed to defining a five-gene score associated with survival after liver resection [11].

Recently, the application of next-generation sequencing (NGS) technologies to the study of the HCC genome and exome is shedding light on the identification of novel driver mutations $[12,13]$. Interestingly, one of the realizations of these NGS studies was that the contribution of pre-mRNA alternative splicing - that is, the alternative inclusion of exons in the mature mRNA - to the repertoire of expressed genes in HCC had been underestimated [12-14]. Indeed, the prominent role of alternative exon selection in gene expression had been previously anticipated by the early results of the ENCODE project [15] and by the use of specialized 'Splice Arrays' platforms across human tissues and cell lines [16]. However, the increasing application of NGS technologies to sequence cDNAs generated by reverse transcription of RNAs, the RNA sequencing technology, has shown that more than $90 \%$ of human introncontaining genes undergo alternative splicing, thus making alternative pre-mRNA splicing to a great extent responsible for the diversity of the transcriptome $[17,18]$. It is therefore plausible that deviations from the physiologically regulated process of alternative splicing can make a significant contribution to disease development, including carcinogenesis [19,20].

The potential functional consequences of alternative splicing not only include the generation of a diverse proteome, with protein variants that can have impaired or even opposing biological roles and different cellular localizations, but also the introduction of premature termination 
codons, which may lead to mRNA downregulation by nonsense-mediated decay (NMD) [21,22]. Most alternative splicing events do not result in a complete change of mRNA isoforms expressed in the cell, but lead to an alteration in the ratios of the isoforms expressed. Although our knowledge of the changes in splicing events and the presence of alternative transcripts in diseased and tumor tissues has markedly increased over the past few years, the biological significance of these alterations is not completely known. It may be that some of these changes are a consequence rather than a cause of the underlying disease. However, direct biochemical evidence on the protumorigenic role of some of these alternatively spliced variants is becoming available. For instance, unequivocal functions in cancer progression have been elucidated for splice variants of the RON tyrosine kinase that favor epithelial-mesenchymal transition, or for the glycolytic enzyme pyruvate kinase, which impaired splicing towards a fetal isoform has been associated with a cancer-specific program $[17,19,22]$.

Pre-mRNA splicing is a complex process directed by signals present in the pre-mRNA sequence (cis-acting elements), executed by small nuclear ribonucleoprotein particles (snRNPs), and regulated by a number of trans-acting protein factors binding to the cis elements in the premRNA sequence. Most alterations in pre-mRNA splicing linked to disease have been attributed to single nucleotide mutations in splice sites, or splice regulatory elements present in exons and introns. Conservative estimations suggest that up to a third of disease-causing polymorphisms could have the potential to disrupt splicing $[19,23]$. Notwithstanding the relevance of these mutations, increasing evidence also points to changes in the expression and activity of transsplicing regulatory proteins in the alterations of pre-mRNA splicing in disease [24-27]. The disruption of normal splicing in liver cancer, and the biological consequences of the expression of tumor-associated splice variants, have been recently reviewed elsewhere [28]. Here, after briefly summarizing the basic mechanisms involved in pre-mRNA splicing and its control, we will discuss some examples of trans-splicing regulatory factors recently involved in the process of hepatocarcinogenesis.

Pre-mRNA splicing \& alternative splicing Introns are noncoding sequences present in practically all eukaryotic pre-mRNAs and account for more than $90 \%$ of the length of these molecules. Introns need to be removed in order to generate the correct concatenation of exons prior to the translation process. Removal of introns is carried out by the spliceosome, a complex molecular machine composed by five types of snRNPs, termed U1, U2, U5 and U4/U6, and a wide array of non-snRNPs splicing factors. The spliceosome needs to recognize consensus sequences at the boundaries of introns, including the $5^{\prime}$ donor splice site beginning with a GU dinucleotide, and the $3^{\prime}$ acceptor splice site ending with an AG dinucleotide. It also carries out the enzymatic process of splicing, which consists in two sequential transesterification reactions. Recognition of the $5^{\prime}$ splice site is carried out by the U1 snRNP, while the $3^{\prime}$ acceptor site, together with the so-called branching sequence that precedes the $3^{\prime}$ acceptor site, are recognized by U2 snRNP and the auxiliary factor U2AF, respectively (Figure 1). Subsequently, the joining of the preassembled U4/U6-U5 snRNPs results in the formation of the catalytically active complex that enables the transesterification reactions, releasing the intron and joining the adjacent exons [29].

Typically, human exons comprise between 50 and 300 nucleotides, with an average length of 134 nucleotides, while introns encompass an average of 3400 nucleotides [22]. The spliceosome has to accurately recognize the abovementioned consensus sequences for the proper excision of introns. When the sequence of these consensus splice sites is not well conserved, the so-called 'weak sites', their affinity for the respective snRNPs is reduced. This fact, together with the presence of pre-mRNA secondary structures and other RNA binding factors, may give rise to the alternative inclusion or exclusion of pre-mRNA segments in the mature mRNA, for example, the process of alternative splicing [27,30]. Alternative splicing needs to be finely tuned to guarantee the proper expression of splice variants in a tissue- and context-dependent fashion, integrating both intracellular and extracellular physiological signals $[17,22]$. The timely recognition of splice sites by the splicing machinery is aided by the previously mentioned trans-factors, which bind to nonsplice site sequences present in exons and introns. The sequences that promote exon recognition by the spliceosome are termed exonic splicing enhancers (ESEs) and intronic splicing enhancers (ISEs), while those that inhibit exon recognition are known as exonic splicing 


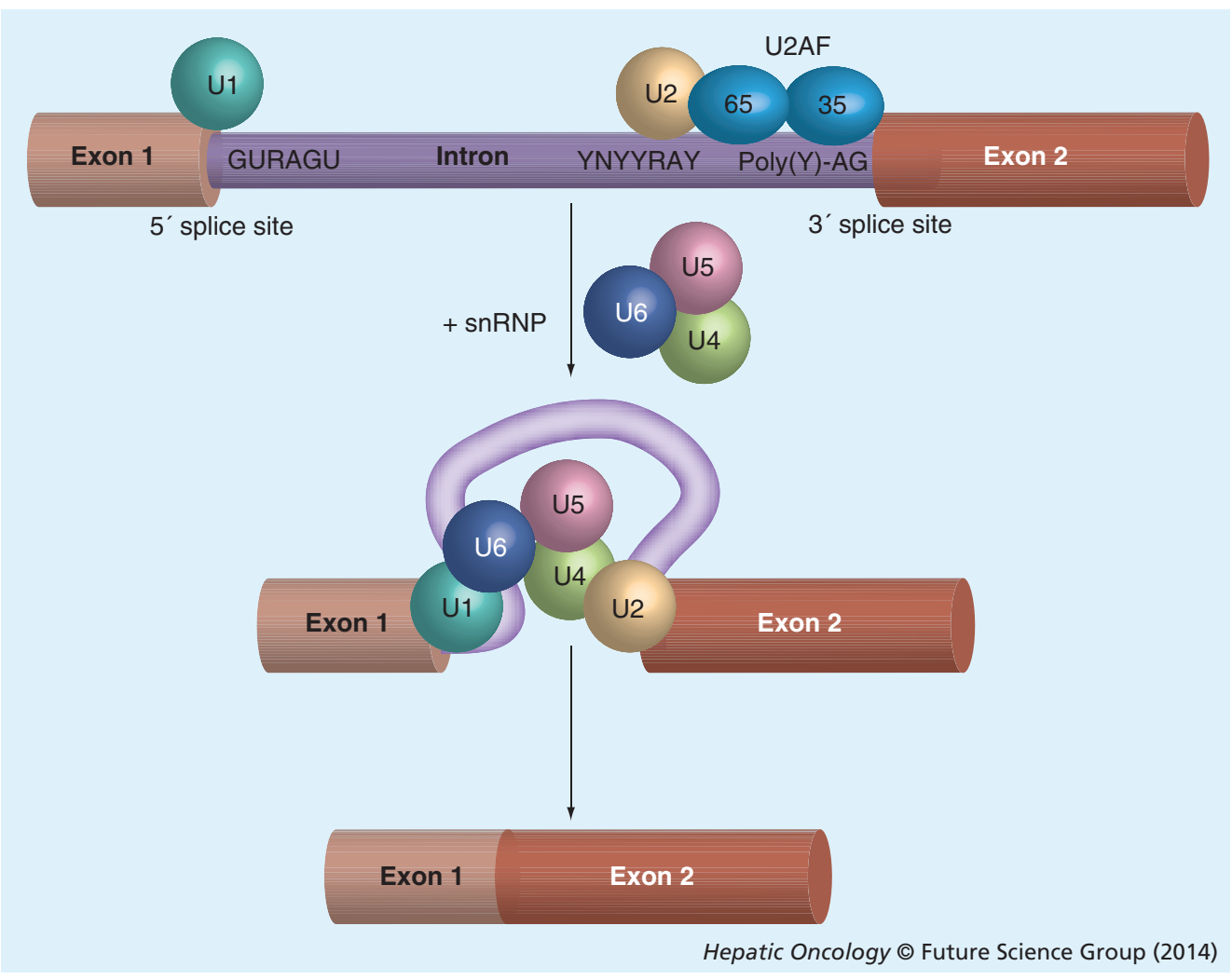

Figure 1. Consensus sequences in pre-mRNA and sequential interaction with small nuclear ribonucleoproteins. Scheme indicating donor and acceptor sites in a eukaryotic gene, as well as the interaction with snRNPs during splicing. The essential elements required for pre-mRNA splicing include the $5^{\prime}$ and $3^{\prime}$ splice sites, the branch point (YNYYRAY) and the polypyrimidine-rich tract $(\operatorname{Poly}(\mathrm{Y}))$ located upstream of the $3^{\prime}$ splice site. The splicing factor U2AF is formed by two subunits that bind to the $3^{\prime}$ splice site and the polypyrimidine tract. U2AF facilitates the binding of U2 snRNA in the U2 snRNP complex to the branch site. The U1 snRNP particle binds to the upstream 5' splice site through base paring of the U1 snRNA. Subsequently, the assembly of the snRNPs U4, U5 and U6 is necessary for the constitution of the spliceosome and the removal of introns. snRNPs: Small nuclear ribonucleoproteins.

silencers (ESSs) and intronic splicing silencers (ISSs) (Figure 2A) [30]. ESEs are particularly abundant within constitutive exons, clustering around splice sites, while ESSs markedly affect splicing by defining exon boundaries when there are multiple or cryptic splice sites, and these elements are believed to play a central role in the regulation of alternative splicing [31]. There are two major groups of trans-regulatory factors that have been characterized in more detail: the serine/arginine-rich (SR) proteins and the heterogeneous nuclear ribonucleoproteins (hnRNPs) [25]. In general, SR proteins facilitate the interaction of snRNPs at splice sites and promote exon inclusion by binding to splicing enhancers. Conversely, hnRNPs favor exon exclusion through their interaction with splicing silencers and the inhibition of the binding and activity of positive splice factors (Figure 2B). However, this view has been recently challenged in various in vivo studies that demonstrated that SR proteins also may participate in exon skipping events, while hnRNPs proteins can also promote exon inclusion [32-34]. These findings add further complexity to the overall process of pre-mRNA splicing and its regulation. There are other factors involved in pre-mRNA splicing regulation that are not included in these two main families, such as the CUGBP1 and SLU7 mRNA-binding proteins; these will be introduced later in this article [35].

hnRNPs are highly abundant proteins in the cell nucleus, and in mammals there are at least 24 members of this family with diverse structures [36]. According to their primary sequence characteristics, 12 polypeptides are included in 
the SR family of proteins, and their complex nomenclature has been recently unified (Figure 2B) [37]. Binding sites for these two families of regulatory proteins with antagonistic effects on splicing can be found in most pre-mRNAs. Hence, even small changes in their expression or biochemical activity can have profound effects on the relative expression of splice variants [38,39]. Next, we will comment on some recent findings on the involvement of SR and hnRNP splicing regulatory factors in hepatocarcinogenesis. However, in spite of the well-known role of these factors in splicing regulation, one should bear in mind that over the past decade their cellular functions have been significantly extended. For instance, SR proteins have been involved in genome stability, chromatin binding and mRNA stability, elongation, export and translation [17,40-42], while hnRNPs have additional roles in
DNA repair, telomere synthesis, and transcription and translation regulation [26,42]. Therefore, impaired regulation of these splicing factors may have profound and manifold effects on cell fate.

\section{Splicing factors in hepatocarcinogenesis}

Abnormal expression of splicing factors can induce the generation of mRNA isoforms that do not exist or are present at very low levels in normal cells. Altered expression, activity and intracellular localization of several splicing regulatory proteins has been reported in connection with carcinogenic processes. In some cases, molecular evidence on the effects of these alterations on the generation of procarcinogenic splice variants has been also provided [17,19,20,39]. Compared with other types of tumors this information is less abundant for liver cancer, although there are accumulating reports that
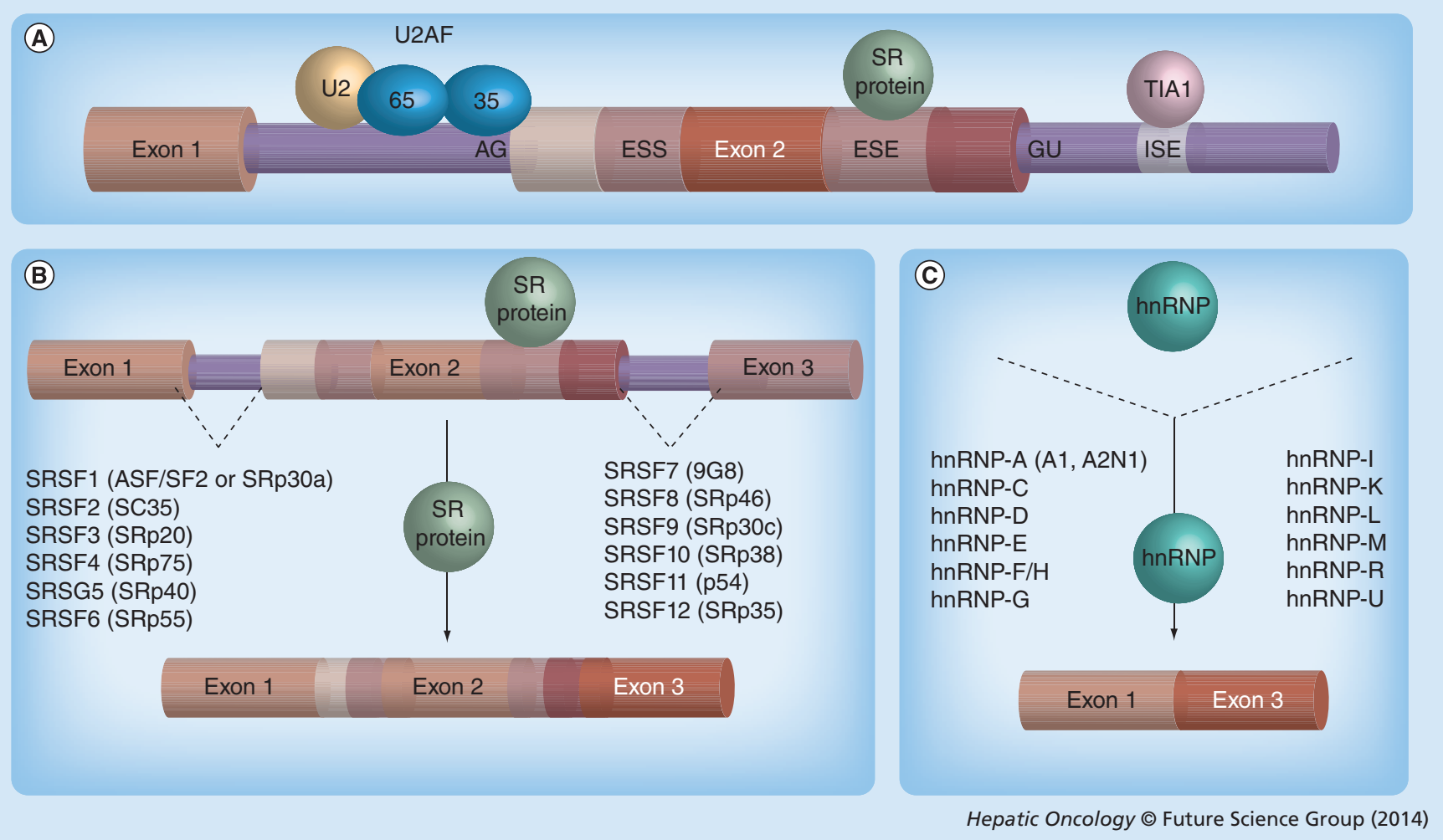

Figure 2. Interaction of splicing regulatory proteins with exonic and intronic regulatory sequences. (A) SR proteins bind to ESES and facilitate the binding of U2AF to weak 3' splice sites. SR proteins also stimulate U1 snRNP binding to a downstream 5' splice site. SR proteins antagonize the negative effect on splicing of hnRNPs that interact with ESSs. In many transcripts, U-rich sequences situated immediately downstream of 5' splice sites, denominated ISEs, are bound by factors such as TIA1 to facilitate U1 binding. (B \& C) Known SR and hnRNPs emphasizing their opposing functions in alternative pre-mRNA splicing. While SR proteins favor exon inclusion, hnRNPs promote exon exclusion.

ESE: Exonic splicing enhancer; ESS: Exonic splicing silencer; hnRNP: Heterogeneous nuclear ribonucleoprotein; ISE: Intronic splicing enhancer; SR: Serine/arginine-rich. 
identify aberrantly spliced genes and some of them analyze the molecular consequences of such variations [28]. Nonetheless, apart from the identification of some splice site mutations, knowledge on the underlying mechanisms leading to the impairment of pre-mRNA splicing is only recently being acquired in liver disease and tumorigenesis.

Among the earliest observations of altered hnRNPs expression in human HCC was an immunohistochemical study that reported increased protein levels of hnRNPH in four HCC tissue specimens compared with normal liver tissues [43]. More recently, another immunohistochemical study showed that hnRNPA2/B1 expression was increased from undetectable levels in normal human liver tissue to a robust positive signal in hepatitis B virus-related HCCs (49 out of 54 samples examined) [43]. This work also examined hnRNPA2/B1 expression in hepatitis tissues (most hepatitis B virus infected), and found a positive signal in all of them, although less intense than in HCCs. Another intriguing finding in this report was the varying subcellular distribution of hnRNPA2/B1, from a nuclear localization in hepatitis samples and well-differentiated HCCs, to a predominantly cytoplasmic staining in poorly differentiated tumors [43]. A similar pattern of expression and subcellular localization of hnRNPA2/B1 along disease progression (from hepatitis to advanced HCC) had been previously observed in other types of tumors, such as lung cancer, also correlating with the advancement of the disease [44]. hnRNPA1 is another member of the hnRNP family frequently overexpressed in cancer [43,45-47]. Its status in HCC was not known until recently, when a thorough study reported a significant upregulation of hnRNPA1 mRNA in human liver tumors (94 HCCs evaluated) [48]. Importantly, hnRNPA1 expression (both mRNA and protein) was higher in HCC tissues from patients with recurrent disease and poorer prognosis [48]. This study also provided mechanistic insights into the consequences of hnRNPA1 upregulation in HCC. In vitro hnRNPA1 expression promoted HCC cell invasion through the generation of the CD44 v6 splice variant [27,48], an isoform of the CD44 membrane glycoprotein and a hyaluronic acid receptor involved in the development and progression of different tumors including HCC [49]. The overexpression of hnRNPH, hnRNPA2/ B1 and hnRNPA1 proteins in human HCC was recently corroborated in another study, which also demonstrated the elevation of their respective mRNA levels in tumor tissues ( $42 \mathrm{HCC}$ samples) [50].

Regarding SR proteins there is also limited information available on their expression in healthy and diseased liver tissues. The previously referred study that addressed the expression levels of hnRNPH, hnRNPA2/B1 and hnRNPA1 in human HCC also showed SF2/ASF (SRSF1) upregulation in liver tumors [50,51]. Interestingly, this work also mentioned that SRSF3 (SRP20) protein was undetectable in HCC tissues [50]. Consistently, we show here that SRSF3 mRNA levels are significantly reduced in HCC tissue samples compared with control liver tissues (Figure 3A). Together, these observations may be highly relevant to the process of hepatocarcinogenesis. Indeed, a recent report demonstrated that hepatocyte-specific deletion of the Srsf3 gene in mice resulted in severely deranged postnatal hepatocellular maturation and differentiation [52]. SRSF3 was shown to be very important for the correct expression of a network of transcription factors involved in liver maturation and function (including HNF1 $\alpha, \mathrm{C} / \mathrm{EBP} \beta$ and FOXA2), as well as for cholesterol-metabolizing genes $[52,53]$. In particular, impaired splicing of $H n f l \alpha$ and Hmgcs 1 was observed in the liver of SRSF3 knockout mice [52,54]. Therefore, the loss of SRSF3 expression could be one important determinant in the multistep process of liver cancer development, in which, as we mentioned at the beginning of this review, hepatocellular dedifferentiation is a hallmark $[2-4,43]$.

Another recent microarray-based gene expression study of liver tissues from normal and obese patients detected reduced mRNA levels of SRSF2, SRSF7 and SRSF10, among other RNAprocessing genes [55]. These findings were reproduced in the liver of mice that were fed a highfat diet. The functional relevance of SRSF10impaired expression was demonstrated upon experimental downregulation of its expression, which resulted in enhanced hepatic lipogenesis, likely through the induction of the $\beta$-splice variant of the Lpin1 gene, a transcriptional regulator of lipid metabolism [55,56]. Reduced expression of SRSF10 may, therefore, be important in the progression of liver disease associated with obesity, a condition recognized as an independent risk factor for HCC development [57]. Nevertheless, in a different study the expression of SRSF4 (SRP75), another SR protein, proved quite stable 


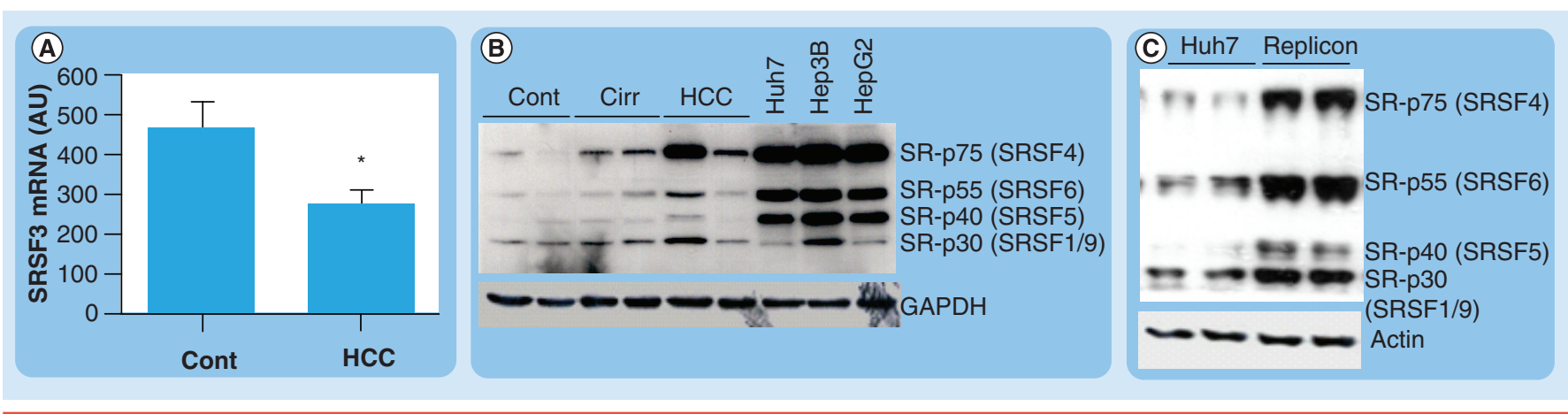

Figure 3. Expression of SRSF3 and phosphorylation of serine/arginine-rich proteins in liver tissues and hepatocellular carcinoma cell lines. (A) Analysis of SRSF3 mRNA levels by quantitative PCR in samples from normal human liver tissues $(n=8)$ and HCC tissues $(n=13)$. mRNA levels of the 36B4 gene were used for normalization. (B) Western blot analysis of basal levels of serine/arginine-rich protein phosphorylation in extracts from cont livers, cirr livers, HCC tissues and the indicated HCC cell lines. Membranes were probed with an antibody specific for a phosphorylated serine/arginine-rich epitope (mAb104), and for GAPDH for a loading control. (C) Western blot analysis of serine/arginine-rich proteins phosphorylation in control and Huh7 cells infected with a hepatitis $C$ virus replicon. The membrane was probed with the mAb104 antibody and with antiactin antibodies for loading control. Tissue samples analyzed in (A) and (B) have been previously described along with the approval of the corresponding ethics review committees [56]. Quantitative PCR and western blot analysis were performed as described [56].

${ }^{*} \mathrm{p}<0.05$.

Cirr: Cirrhotic; Cont: Control; HCC: Hepatocellular carcinoma.

when control human liver tissues where compared with those from patients with hepatitis $\mathrm{C}$ virus (HCV)-induced liver cirrhosis, dysplasia, and early and advanced HCC [58]. Similarly, hnRNPF mRNA and protein levels appeared unaltered in HCC in comparison with nontumoral liver tissues [50]. Taken together, these observations indicate that not all splicing regulatory factors are systematically affected in liver disease, suggesting that those with an impaired expression (induced or decreased) could indeed play a specific role in liver pathology.

\section{Cell signaling pathways regulate splicing} factors in HCC

An important aspect that has developed over the past decade is the influence of extracellular stimuli on the pre-mRNA splicing process $[27,28,39,59,60]$. Signaling pathways can modulate the activity, stability, expression and intracellular localization of splicing regulatory proteins of the hnRNP and SR families, as well as other splice regulators such as SLU7 and CUGBP1 $[35,48,61,62]$. One of the most studied signalingrelated post-translational modifications of these proteins is their reversible phosphorylation [48,51,63], although other covalent modifications, such as methylation, acetylation and ubiquitylation, are emerging as important regulatory mechanisms [27,50,51]. In SR proteins extensive phosphorylation on serine residues in the SR domain can modulate their subcellular distribution and interactions [27]. It is sequentially performed by the SR protein kinases SRPK1 and SRPK2, and other protein kinases such as PKB/AKT, and a family of nuclear CDC2-like kinases [51]. These kinases can be triggered by growth factors and cytokines upon binding to their membrane receptors, thus, relaying signals from the extracellular environment to the splicing machinery $[39,50,51,60]$. Interestingly, compared with control liver tissues, we observed increased phosphorylation of specific SR proteins in liver samples obtained from cirrhotic patients and HCC tissues (Figure 3B). This modification was much more extensive in HCC cell lines (Figure 3B), and was markedly potentiated when Huh7 cells were infected with a subgenomic replicon derived from the HCV (Figure 3C) [64], suggesting a broad impact of HCV infection on pre-mRNA splicing in the liver. In the context of chronic liver disease induced by viral infection, alcohol abuse or metabolic derangements, the hepatic extracellular milieu is a very reactive environment rich in inflammatory cytokines and growth factors, believed to play a central role in HCC development [65]. The identity of the factors and signaling pathways responsible for the enhanced phosphorylation of SR proteins illustrated here, and for the alterations in the expression of splicing regulators in liver disease reviewed above, is not completely known. 
In the past few years EGFR-triggered signaling has been identified as a very effective mechanism in the regulation of alternative pre-mRNA splicing in different cellular backgrounds. For instance, EGF was shown to modulate the splicing of the CD44 cell surface protein through the Ras/MAPK cascade in HeLa cells, and this response was important for cell cycle progression [66]. More recently, and with the application of high-throughput sequencing techniques, it was found that EGF treatment of HEK-293 cells induced profound changes in splicing of endogenous genes, and these effects were mediated through a newly defined AKT-SRPK-SR axis emanating from the EGFR [67]. In another recent study the expression of the constitutively active EGFRvIII mutant, which is frequently found in glioblastoma cells, was demonstrated to promote tumor growth and metabolic reprogramming to a glycolytic phenotype, in part through its effects on pre-mRNA splicing [53]. The presence of the EGFRvIII mutant significantly upregulated hnRNPA1 gene expression and the increased levels of this splicing regulator resulted in the generation of an alternatively spliced form of the Myc-interacting protein Max, an enhancer of Myc-dependent cell transformation [53].

Regarding HCC cells, the first detailed report on the potential role of EGFR downstream signaling focused on the alternative splicing of the $K L F 6$ gene, a zinc finger transcription factor and a tumor suppressor [54]. KLF6 pre-mRNA can be alternatively spliced into three variants; one of them, known as KLFG SV1, lacks the DNA-binding domain and can behave as a dominant-negative protein, interfering with the full length KLF6 protein [28]. In this study it was observed that EGFR-mediated activation of the Ras/PI3K/AKT cascade led to the generation of the dominant-negative KLF6 SV1, and promoted enhanced HCC cell proliferation. The authors could identify the SR protein ASF/SF2 (SRSF1) as an essential mediator of $K L F 6$ alternative splicing in the face of EGFR activation [54]. Shortly after, another study from our group identified a signaling pathway starting also at the EGFR that modulated the splicing of another tumor suppressor, the $p 73$ gene [56]. In this case EGFR activation led to the upregulation of a p73 splice variant lacking exon $2(\Delta \mathrm{Ex} 2 \mathrm{p} 73)$, which behaves as a dominant negative inhibitor not only for full-length p73 but also for the closely related tumor suppressor gene $\mathrm{p} 53$, and displays protumorigenic properties [68]. Mechanistically it was shown that EGFR effects on p73 alternative splicing were dependent on JNK1 signaling. EGFR-mediated JNK1 activation led to the transcriptional downregulation of $S L U 7$ gene expression [62]. SLU7 is a splicing factor involved in the correct selection of the $3^{\prime}$ site during the second step of splicing, therefore its reduced availability can result in exon skipping, the mechanism responsible for the generation of the $\Delta \mathrm{Ex} 2 \mathrm{p} 73$ variant [56]. Such a possibility was confirmed upon SLU7 knockdown in HCC cells, a manipulation that promoted the increase in $\Delta \mathrm{Ex} 2 \mathrm{p} 73$ transcripts in the absence of EGFR stimulation [56]. This work provided two further relevant pieces of information; first, the identification of the EGFR ligand, AR, as a key autocrine driver of the EGFR/JNK1 axis leading to SLU7 repression and $\Delta \mathrm{Ex} 2 \mathrm{p} 73$ upregulation and; second, the fact that reduced SLU7 expression and enhanced p73 splicing into its protumorigenic $\Delta \mathrm{Ex} 2 \mathrm{p} 73$ form was found not only in HCC tissues but also in the liver of cirrhotic patients [54].

The important role of the EGFR signaling system in the deregulation of pre-mRNA alternative splicing in HCC cells has been recently strengthened by another interesting report [50]. This work found that the insulin receptor $\alpha$-chain splice variant A (IR-A), which is normally expressed in fetal tissues was upregulated in HCC tissues and HCC cell lines. This IR-A splice variant lacks exon 11 and shows ligand affinities and kinetic properties that are at variance with the full length IR-B splice form, which is present in mature insulin-responsive tissues, and allow the transmission of survival and proliferation signals [69]. This switch in the relative amounts of IR isoforms is pathologically meaningful given the accumulating evidence linking hyperinsulinemia, insulin resistance and obesity with the risk of HCC development [57]. It was found that EGFR activation of the MEK/ERK pathway in HCC cells led to the upregulation of a series of splice regulatory proteins, including hnRNPH, hnRNPA1 and CUGBP1, and that these factors contributed to the generation of the IR-A splice variant [52]. Interestingly, siRNA-mediated knockdown of the EGFR, or its pharmacological inhibition, resulted in the downregulation of the abovementioned splicing factors and also attenuated the expression of the IR-A variant. Moreover and as previously reported for the splicing of p73 into its $\Delta \mathrm{Ex} 2 \mathrm{p} 73$ form [56], the constitutively high levels of IR-A present in HCC cells were 
dependent in part on the expression of the EGFR ligand AR, likely through the activation of an AR/EGFR autocrine loop [52].

Taken together, these reports uncover a central role for the EGFR signaling system in the control of alternative pre-mRNA splicing in HCC cells (Figure 4). The nature of the genes so far identified as splice targets downstream of the EGFR, two tumor suppressor genes (KLFG and $p 73$ ) and a membrane receptor that conveys prosurvival and mitogenic signals $(I R)$, suggests that these splicing events may be biologically relevant. Although the EGFR can be bound and activated by a variety of ligands, a predominant role for AR seems to emerge from these studies. $\mathrm{AR}$ expression is very low in the healthy liver, however, it is induced during parenchymal inflammation and injury, contributing to tissue repair but also to liver disease progression $[52,65,70]$. In liver cancer persistent AR expression maintains a constitutive autocrine loop contributing to HCC cell survival and proliferation, and has been also identified as a critical factor in HCC resistance to standard and targeted chemotherapeutics [71,72]. Alterations in pre-mRNA splicing may thus represent an additional procarcinogenic mechanism driven by the AR/EGFR axis.

As could be expected for a molecularly heterogeneous tumor like HCC the EGFR signaling system is not alone in the control of alternative splicing by the tumor microenvironment. HGF, a potent mediator of liver regeneration and repair also involved in hepatocarcinogenesis, is produced by mesenchymal cells and activates its cognate receptor c-MET, present in epithelial cells such as hepatocytes [52,73,74]. The HGF/c-MET system has been recently identified as an additional regulator of the alternative splicing of the KLF6 tumor suppressor gene into its SV1 variant, which is increasingly recognized as a protumorigenic splice form in the liver [75]. In this case, the authors found that two SR proteins, SRSF3 and SRSF1, in a complex interaction were involved in the regulation of KLF6 alternative splicing in HCC cells [76]. HGF stimulation of the PI3K/ AKT pathway led to reduced SRSF3 protein levels, which in turn had important consequences

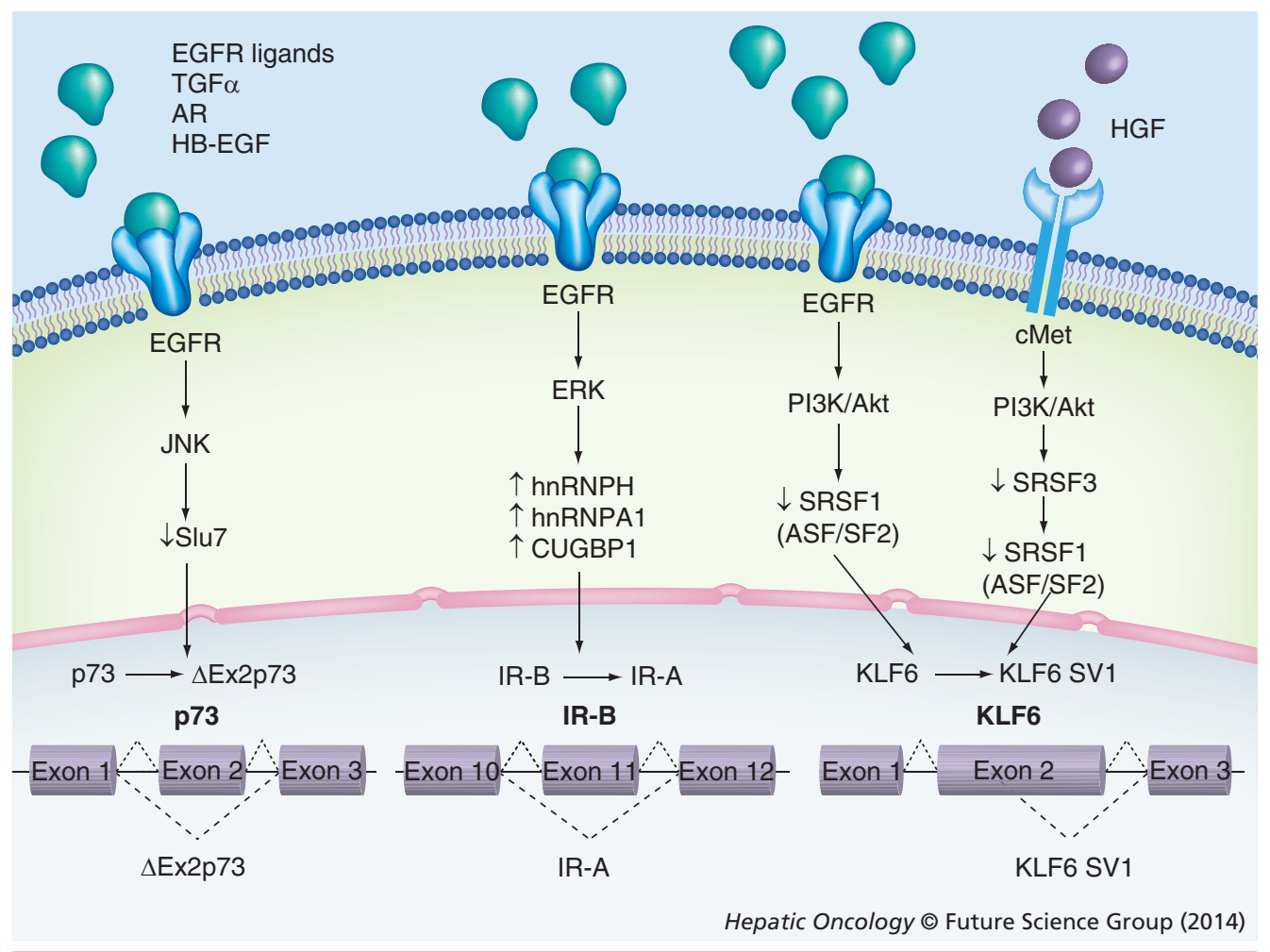

Figure 4. Regulation of pre-mRNA alternative splicing by growth factor-mediated signaling in hepatocellular carcinoma cells. Intracellular pathways and splice regulatory proteins involved in the alternative splicing of $p 73, I R$ and $K L F 6$ transcripts induced by EGFR and HGF receptor cMet activation. In addition to EGF, ligands of the EGFR include TGF $\alpha$, AR and HB-EGF.

$\uparrow$ : Increase; $\downarrow$ : Decrease; HB-EGF: Heparin-binding EGF. 
for the expression of SRSF1. It was observed that reduced SRSF3 availability altered $S R S F 1$ pre$\mathrm{mRNA}$ splicing, leading to the generation of a $S R S F 1$ transcript in which the natural termination codon is recognized as a premature stop codon and the mRNA is degraded by the NMD pathway [21]. Reduced SRSF1 protein levels were shown in turn to enhance $K L F 6$ splicing into its SV1 form, altering the $S V 1 / K L F G$ ratio (Figure 4) [76]. Nevertheless, the enhanced alternative splicing of KLFG into its SV1 variant, frequently found in HCC tissues [75], is likely to involve additional mechanisms besides the one involving HGF-mediated SRSF1 downregulation, since this $S R$ protein is frequently found upregulated in epithelial tumors, including HCC [50,77]. In addition to the identification of HGF-mediated regulation of $K L F G$ alternative splicing, this study is probably the first example of alternative splicing-coupled NMD of pre-mRNA processing factors in HCC cells. Alternative splicing-coupledNMD is frequent among SR proteins, which can regulate their own expression as well as that of other RNA-binding proteins through the generation of NMD-prone splice variants [21,27,55]. This work and the abovementioned studies illustrate the complexity of the pathways and interactions triggered by extracellular signaling molecules that lead to changes in alternative splicing of pre-mRNAs in HCC cells. Nevertheless, the situations described herein are likely to be an oversimplification of what is really happening in tumor tissues, where an intricate network of signals from soluble factors, matrix components and cellular interactions act in concert.

\section{Future perspective}

The increased application of NGS techniques to the analysis of gene expression, including the recent findings of the ENCODE project [78], has led to the realization of the enormous influence of alternative pre-mRNA splicing on the repertoire of proteins expressed in a cell or tissue. Consequently, the role of impaired pre-mRNA splicing in disease, although generally accepted, may have been underestimated. The detailed characterization of the molecular mechanisms leading to the control of normal and pathological pre-mRNA splicing, as well as the demonstration of the disease-promoting capacity of the identified splice variants, is a research line that should be pursued. This is particularly relevant in a molecularly complex tumor such as HCC. Hand in hand with these studies, novel strategies aimed at the modulation of alternative splicing are emerging [19,79]. A better knowledge of the alterations in premRNA splicing triggered by signaling pathways involved in hepatocarcinogenesis will help to understand the mechanisms of this disease, its resistance to targeted therapies and to foster the development of more efficacious treatments.

Financial \& competing interests disclosure

This work was supported by the agreement between FIMA and the 'UTE project CIMA'; RTICC-RD06 00200061 (C Berasain, MA Avila); CIBEREhd (I Uriarte, J Prieto, C Berasain, MA Avila); FIS PI10/02642, PI10/00038, FIS PI13/00385 and PI13/00359 (C Berasain, MA Avila) from Instituto de Salud Carlos III. MU Latasa and R Urtasun were supported by a 'Ramón y Cajal' and a 'Torres Quevedo' contract from Ministerio de Educación, respectively. M Elizalde was supported by a fellowship from Gobierno de Navarra. O García-Irigoyen was supported by a FPU fellowship from Ministerio de Educación, Cultura $y$ Deporte, Spain. The authors have no other relevant affiliations or financial involvement with any organization or entity with a financial interest in or financial conflict with the subject matter or materials discussed in the manuscript apart from those disclosed.

No writing assistance was utilized in the production of this manuscript.

\section{References}

Papers of special note have been highlighted as:

- of interest

-1 of considerable interest

1 Trevisani F, Cantarini MC, Wands JR, Bernardi M. Recent advances in the natural history of hepatocellular carcinoma. Carcinogenesis 29(7), 1299-1305 (2008).

2 Avila MA, Berasain C, Torres L et al. Reduced mRNA abundance of the main enzymes involved in methionine metabolism in human liver cirrhosis and hepatocellular carcinoma. J. Hepatol. 33(6), 907-914 (2000).

3 Berasain C, Herrero J-I, García-Trevijano ER et al. Expression of Wilms' tumor suppressor in the liver with cirrhosis: relation to hepatocyte nuclear factor 4 and hepatocellular function. Hepatology 38(1), 148-157 (2003).

4 Hoshida Y, Villanueva A, Kobayashi M et al. Gene expression in fixed tissues and outcome in hepatocellular carcinoma. N. Engl. J. Med. 359(19), 1995-2004 (2008).
5 Han Z-G. Functional genomic studies: insights into the pathogenesis of liver cancer. Annu. Rev. Genomics Hum. Genet. 13, 171-205 (2012).

6 Calvisi DF, Frau M, Tomasi ML, Feo F, Pascale RM. Deregulation of signalling pathways in prognostic subtypes of hepatocellular carcinoma: novel insights from interspecies comparison. Biochim. Biophys. Acta. 1826(1), 215-237 (2012).

Nault J-C, Zucman-Rossi J. Genetics of hepatobiliary carcinogenesis. Semin. Liver Dis. 31(2), 173-187 (2011). 
8 Woo HG, Park ES, Lee J-S et al. Identification of potential driver genes in human liver carcinoma by genomewide screening. Cancer Res. 69(9), 4059-4066 (2009).

9 Villanueva A, Hernandez-Gea V, Llovet JM. Medical therapies for hepatocellular carcinoma: a critical view of the evidence. Nat. Rev. Gastroenterol. Hepatol. 10(1), 34-42 (2013).

10 Villanueva A, Llovet JM. Targeted therapies for hepatocellular carcinoma. Gastroenterology 140(5), 1410-1426 (2011).

11 Nault J-C, De Reyniès A, Villanueva A et al. A hepatocellular carcinoma 5-gene score associated with survival of patients after liver resection. Gastroenterology 145(1), 176-187 (2013).

12 Li S, Mao M. Next generation sequencing reveals genetic landscape of hepatocellular carcinomas. Cancer Lett. 340(2), 247-253 (2012).

13 Nakagawa H, Shibata T. Comprehensive genome sequencing of the liver cancer genome. Cancer Lett. 340(2), 234-240 (2012).

14 Huang Q, Lin B, Liu H et al. RNA-seq analyses generate comprehensive transcriptomic landscape and reveal complex transcript patterns in hepatocellular carcinoma. PLoS ONE 6(10), e26168 (2011).

15 Tress ML, Martelli PL, Frankish A et al. The implications of alternative splicing in the ENCODE protein complement. Proc. Natl Acad. Sci. USA 104(13), 5495-5500 (2007).

16 Zhou W, Calciano MA, Jordan $\mathrm{H}$ et al. High resolution analysis of the human transcriptome: detection of extensive alternative splicing independent of transcriptional activity. BMC Genet. 10, 63 (2009).

17 Kalsotra A, Cooper TA. Functional consequences of developmentally regulated alternative splicing. Nat. Rev. Genetics. 12(10), 715-729 (2011).

18 Feng H, Qin Z, Zhang X. Opportunities and methods for studying alternative splicing in cancer with RNA-seq. Cancer Lett. 340(2), 179-191 (2012).

19 Singh RK, Cooper TA. Pre-mRNA splicing in disease and therapeutics. Trends Mol. Med. 18(8), 472-482 (2012).

20 Kaida D, Schneider-Poetsch T, Yoshida M. Splicing in oncogenesis and tumor suppression. Cancer Sci. 103(9), 1611-1616 (2012).

21 Bhuvanagiri M, Schlitter AM, Hentze MW, Kulozik AE. NMD: RNA biology meets human genetic medicine. Biochem. J. 430(3), 365-377 (2010).
22 Kelemen O, Convertini P, Zhang Z et al. Function of alternative splicing. Gene 514(1), 1-30 (2013).

23 Wang G-S, Cooper TA. Splicing in disease: disruption of the splicing code and the decoding machinery. Nat. Rev. Genetics 8(10), 749-761 (2007).

24 Grosso AR, Martins S, Carmo-Fonseca M. The emerging role of splicing factors in cancer. EMBO Rep. 9(11), 1087-1093 (2008).

25 Busch A, Hertel KJ. Evolution of SR protein and hnRNP splicing regulatory factors. WIRES RNA 3(1), 1-12 (2012).

26 Han SP, Tang YH, Smith R. Functional diversity of the hnRNPs: past, present and perspectives. Biochem. J. 430(3), 379-392 (2010).

27 Risso G, Pelisch F, Quaglino A, Pozzi B, Srebrow A. Regulating the regulators: serine/ arginine-rich proteins under scrutiny. IUBMB Life 64(10), 809-816 (2012).

28 Berasain C, Goñi S, Castillo J, Latasa MU, Prieto J, Avila MA. Impairment of premRNA splicing in liver disease: mechanisms and consequences. World J. Gastroenterol. 16(25), 3091-3102 (2010).

29 Will CL, Lührmann R. Spliceosome structure and function. Cold Spring Harbor Persp. Biol. 3(7) (2011).

30 Black DL. Mechanisms of alternative premessenger RNA splicing. Annu. Rev. Biochem. 72, 291-336 (2003).

31 Jensen CJ, Oldfield BJ, Rubio JP. Splicing, cis genetic variation and disease. Biochem. Soc. Trans. 37(Pt 6), 1311-1315 (2009).

32 Talukdar I, Sen S, Urbano R, Thompson J, Yates JR, Webster NJG. hnRNP A1 and hnRNP F modulate the alternative splicing of exon 11 of the insulin receptor gene. PLoS ONE 6(11), e27869 (2011).

33 Huelga SC, Vu AQ, Arnold JD et al. Integrative genome-wide analysis reveals cooperative regulation of alternative splicing by hnRNP proteins. Cell Rep. 1(2), 167-178 (2012).

34 Pandit S, Zhou Y, Shiue L et al. Genomewide analysis reveals $S R$ protein cooperation and competition in regulated splicing. Mol. Cell. 50(2), 223-235 (2013).

35 Dasgupta T, Ladd AN. The importance of CELF control: molecular and biological roles of the CUG-BP, Elav-like family of RNAbinding proteins. WIRES RNA 3(1), 104-121 (2012).

36 Martinez-Contreras R, Cloutier P, Shkreta L, Fisette J-F, Revil T, Chabot B. hnRNP proteins and splicing control. Adv. Exp. Med. Biol. 623, 123-147 (2007).

37 Manley JL, Krainer AR. A rational nomenclature for serine/arginine-rich protein splicing factors (SR proteins). Genes Dev. 24(11), 1073-1074 (2010).

38 Chen M, David CJ, Manley JL. Concentration-dependent control of pyruvate kinase $\mathrm{M}$ mutually exclusive splicing by hnRNP proteins. Nat. Struct. Mol. Biol. 19(3), 346-354 (2012).

39 David CJ, Manley JL. Alternative pre-mRNA splicing regulation in cancer: pathways and programs unhinged. Genes Dev. 24(21), 2343-2364 (2010).

40 Long JC, Caceres JF. The SR protein family of splicing factors: master regulators of gene expression. Biochem. J. 417(1), 15-27 (2009).

41 Twyffels L, Gueydan C, Kruys V. Shuttling SR proteins: more than splicing factors. FEBS J. 278(18), 3246-3255 (2011).

42 Kornblihtt AR, Schor IE, Allo M, Dujardin G, Petrillo E, Munoz MJ. Alternative splicing: a pivotal step between eukaryotic transcription and translation. Nat. Rev. Mol. Cell. Biol. 14(3), 153-165 (2013).

43 Cui H, Wu F, Sun Y, Fan G, Wang Q. Upregulation and subcellular localization of hnRNP A2/B1 in the development of hepatocellular carcinoma. BMC Cancer 10, 356 (2010).

44 Man YG, Martinez A, Avis IM et al. Phenotypically different cells with heterogeneous nuclear ribonucleoprotein A2/ B1 overexpression show similar genetic alterations. Am. J. Respir. Cell Mol. Biol. 23(5), 636-645 (2000).

45 Pino I, Pío R, Toledo G et al. Altered patterns of expression of members of the heterogeneous nuclear ribonucleoprotein (hnRNP) family in lung cancer. Lung Cancer 41(2), 131-143 (2003).

46 Li S, Xu H, Ding H et al. Identification of an aptamer targeting hnRNP A1 by tissue slidebased SELEX. J. Pathol. 218(3), 327-336 (2009).

47 Ushigome M, Ubagai T, Fukuda $\mathrm{H}$ et al. Up-regulation of hnRNP A1 gene in sporadic human colorectal cancers. Int. J. Oncol. 26(3), 635-640 (2005).

48 Zhou Z-J, Dai Z, Zhou S-L et al. Overexpression of HnRNP A1 promotes tumor invasion through regulating CD44v6 and indicates poor prognosis for hepatocellular carcinoma. Int. J. Cancer. 132(5), 1080-1089 (2013).

- Detailed description of the overexpression of a splicing regulator, hnRNP A1, in 
hepatocellular carcinoma (HCC) tissues and cell lines and its contribution to malignancy.

49 Endo K, Terada T. Protein expression of CD44 (standard and variant isoforms) in hepatocellular carcinoma: relationships with tumor grade, clinicopathologic parameters, $\mathrm{p} 53$ expression, and patient survival. J. Hepatol. 32(1), 78-84 (2000).

50 Chettouh H, Fartoux L, Aoudjehane L et al. Mitogenic insulin receptor-A is overexpressed in human hepatocellular carcinoma due to EGFR-mediated dysregulation of RNA splicing factors. Cancer Res. 73(13), 3974-3986 (2013).

- Identification of the EGFR signaling system as a key inducer of the expression of a broad range of splicing factors dysregulated in HCC tissues and cells.

51 Edmond V, Moysan E, Khochbin S et al. Acetylation and phosphorylation of SRSF2 control cell fate decision in response to cisplatin. EMBO J. 30(3), 510-523 (2011).

52 Sen S, Jumaa H, Webster NJG. Splicing factor SRSF3 is crucial for hepatocyte differentiation and metabolic function. Nat. Commun. 4, 1336 (2013).

- First in vivo study demonstrating the pathological consequences of hepatocytespecific deletion of a splicing regulator, SRSF3, in the normal mouse.

53 Babic I, Anderson ES, Tanaka K et al. EGFR mutation-induced alternative splicing of Max contributes to growth of glycolytic tumors in brain cancer. Cell Metab. 17(6), 1000-1008 (2013).

54 Yea S, Narla G, Zhao X et al. Ras promotes growth by alternative splicing-mediated inactivation of the KLF6 tumor suppressor in hepatocellular carcinoma. Gastroenterology 134(5), 1521-1531 (2008).

- First report on the effects of EGFR-mediated cell signaling on the activity of splicing factors in HCC cells.

55 Pihlajamäki J, Lerin C, Itkonen P et al. Expression of the splicing factor gene SFRS10 is reduced in human obesity and contributes to enhanced lipogenesis. Cell Metab. 14(2), 208-218 (2011).

- - Splicing factors are found downregulated in the liver of obese humans and mice. Srsf10 heterozygous mice display impaired lipid metabolism in the liver.

56 Castillo J, Goñi S, Latasa MU et al. Amphiregulin induces the alternative splicing of p73 into its oncogenic isoform DeltaEx2p73 in human hepatocellular tumors. Gastroenterology 137(5), 1805-15. e1-4 (2009).
- First description of an EGFR ligand as an endogenous regulator of alternative splicing in HCC cells.

57 Baffy G, Brunt EM, Caldwell SH. Hepatocellular carcinoma in non-alcoholic fatty liver disease: an emerging menace. J. Hepatol. 56(6), 1384-1391 (2012).

58 Waxman S, Wurmbach E. De-regulation of common housekeeping genes in hepatocellular carcinoma. BMC Genomics 8 , 243 (2007).

59 Shin C, Manley JL. Cell signalling and the control of pre-mRNA splicing. Nat. Rev. Mol. Cell. Biol. 5(9), 727-738 (2004).

60 Blaustein M, Pelisch F, Srebrow A. Signals, pathways and splicing regulation. Int. J. Biochem. Cell. Biol. 39(11), 2031-2048 (2007).

61 Heyd F, Lynch KW. Degrade, move, regroup: signaling control of splicing proteins. Trends Biochem. Sci. 36(8), 397-404 (2011).

62 Shomron N, Alberstein M, Reznik M, Ast G. Stress alters the subcellular distribution of hSlu7 and thus modulates alternative splicing. J. Cell Sci. 118(Pt 6), 1151-1159 (2005).

63 Stamm S. Regulation of alternative splicing by reversible protein phosphorylation. J. Biol. Chem. 283(3), 1223-1227 (2008).

64 Ikeda M, Yi M, Li K, Lemon SM. Selectable subgenomic and genome-length dicistronic RNAs derived from an infectious molecular clone of the HCV-N strain of hepatitis C virus replicate efficiently in cultured Huh7 cells. J. Virol. 76(6), 2997-3006 (2002).

65 Berasain C, Castillo J, Perugorría MJ, Latasa MU, Prieto J, Avila MA. Inflammation and liver cancer: new molecular links . Ann. NY Acad. Sci. 1155, 206-221 (2009).

66 Cheng C, Yaffe MB, Sharp PA. A positive feedback loop couples Ras activation and CD44 alternative splicing. Genes Dev. 20(13), 1715-1720 (2006).

67 Zhou Z, Qiu J, Liu W et al. The Akt-SRPK$S R$ axis constitutes a major pathway in transducing EGF signaling to regulate alternative splicing in the nucleus. Mol. Cell. 47(3), 422-433 (2012).

- Detailed molecular delineation of an intracellular signaling cascade triggered by the EGFR leading to the regulation of serine/arginine-rich protein phosphorylation and splicing regulation.

68 Oswald C, Stiewe T. In good times and bad: p73 in cancer. Cell Cycle 7(12), 1726-1731 (2008).
69 Knudsen L, De Meyts P, Kiselyov VV. Insight into the molecular basis for the kinetic differences between the two insulin receptor isoforms. Biochem. J. 440(3), 397-403 (2011).

70 Berasain C, Perugorría MJ, Latasa MU et al. The epidermal growth factor receptor: a link between inflammation and liver cancer. Exp. Biol. Med. (Maywood) 234(7), 713-725 (2009).

71 Castillo J, Erroba E, Perugorría MJ et al. Amphiregulin contributes to the transformed phenotype of human hepatocellular carcinoma cells. Cancer Res. 66(12), 6129-6138 (2006).

72 Blivet-Van Eggelpoël M-J, Chettouh H, Fartoux L et al. Epidermal growth factor receptor and HER-3 restrict cell response to sorafenib in hepatocellular carcinoma cells. J. Hepatol. 57(1), 108-115 (2012).

73 Michalopoulos GK. Principles of liver regeneration and growth homeostasis. Compr Physiol. 3(1), 485-513 (2013).

74 Goyal L, Muzumdar MD, Zhu AX. Targeting the HGF/c-MET pathway in hepatocellular carcinoma. Clin. Cancer Res. 19(9), 23102318 (2013).

75 Vetter D, Cohen-Naftaly M, Villanueva A et al. Enhanced hepatocarcinogenesis in mouse models and human hepatocellular carcinoma by coordinate KLF6 depletion and increased messenger RNA splicing. Hepatology 56(4), 1361-1370 (2012).

76 Muñoz U, Puche JE, Hannivoort R, Lang UE, Cohen-Naftaly M, Friedman SL. Hepatocyte growth factor enhances alternative splicing of the Kruppel-like factor 6 (KLF6) tumor suppressor to promote growth through SRSF1. Mol. Cancer Res. 10(9), 1216-1227 (2012).

-n Identification of the HGF/c-MET signaling system as a novel regulator of the expression and activity of SRSF3 and SRSF1 splicing factors in HCC cells.

77 Anczuków O, Rosenberg AZ, Akerman M et al. The splicing factor SRSF1 regulates apoptosis and proliferation to promote mammary epithelial cell transformation. Nat. Struct. Mol. Biol. 19(2), 220-228 (2012).

78 Bernstein BE, Birney E, Dunham I et al.; ENCODE Project Consortium. An integrated encyclopedia of DNA elements in the human genome. Nature 489(7414), 57-74 (2012).

79 Spitali P, Aartsma-Rus A. Splice modulating therapies for human disease. Cell 148(6), 1085-1088 (2012). 